\title{
Bending Instability of an Accretion Disc Around a Black Hole
}

\author{
D. Molteni ${ }^{1}$, K. Acharya ${ }^{2}$, Oleg Kuznetsov ${ }^{3}$, D. Bisikalo ${ }^{4}$, Sandip K. Chakrabarti ${ }^{5,2}$ \\ ${ }^{1}$ Department of Physics, University of Palermo, Palermo, Italy \\ ${ }^{2}$ Centre for Space Physics, 114/v/1A Raja S.C. Mullick Rd., Kolkata 700047 \\ ${ }^{3}$ Keldysh Institute of Applied Mathematics, Moscow \\ ${ }^{4}$ Institute of Astronomy, Moscow \\ ${ }^{5,2}$ S. N. Bose National Centre for Basic Sciences, JD-Block, Salt Lake, Kolkata, 700098, INDIA \\ e-mail: molteni@gifco.fisica.unipa.it,space_phys@vsnl.com,kuznecov@spp.keldysh.ru,bisikalo@inasan.rssi.ru\& \\ chakraba@boson.bose.res.in
}

\begin{abstract}
We demonstrate that generically an accretion disk around a compact object could have a new type of instability in that the accretion flow need not be symmetric with respect to the equatorial plane even when matter is supplied symmetrically farther out. We find that this behaviour is mainly due to interaction of outgoing matter bounced off the centrifugal barrier and the incoming accretion. We believe that X-ray variability could be developed by this instability.
\end{abstract}

Subject headings: accretion, accretion disks — black hole physics — hydrodynamics — Instabilities — shock waves

Accepted for publication in ApJ Letters 


\section{Introduction}

Accretion onto a black hole is generally assumed to be thin and axisymmetric (e.g. Pringle, 1981). In presence of a close companion, accretion disk can develop non-axisymmetric instability and spiral shocks are produced (Sawada, Matsuda \& Hachisu, 1986). Bending waves and warps are known to be produced in planetary rings (Greenberg \& Brahic, 1990) and galactic disks (e.g., Pringle, 1997). These instabilities are due to self-gravity of the disk or due to gravity of a nearby companion. In the present Letter, we demonstrate that an accretion disk could develop a new kind of instability, because of interaction between accreting matter and winds and outflows generated from the star or the accretion disk. The interface between the two becomes unstable due to shear instability or Kelvin-Helmholtz instability. In presence of rotation, which is invariably true in the case of an accretion disk, the instability is not the same in both the upper-half and the lower-half. As a result, the accretion process may not be symmetric with respect to the equatorial plane. The instability is present independent of polytropic index or cooling mechanism in the flow.

In the past, a large number of numerical simulations which concern accretion flows around black holes, have been presented in the literature (e.g., Hawley, Wilson \& Smarr 1984; Eggum, Coroniti \& Katz, 1985; Molteni, Lanzafame \& Chakrabarti, 1994; Molteni, Sponholz \& Chakrabarti, 1996; hereafter MSC96 Ryu, Molteni \& Chakrabarti, 1997; Igumenshchev, Abramowicz, Narayan, 2000). These work assumed a priori that the flow has a equatorial symmetry and therefore the flow behaviour was studied on one half and used the 'reflection' boundary condition on the equatorial plane. With the advent of faster computational techniques and computers, one could perform numerical simulations in more realistic cases, namely, in both the halves simultaneously without using such an artificial boundary condition. MSC96 studied oscillating shocks to explain QuasiPeriodic Oscillations, but these oscillations are generated only in a part of the parameter space. The vertical oscillation of the disk we report here occurs in a much larger range of the disk parameters, only amplitude and frequency vary. For very large energy or very low angular momentum when the infall velocity is high, but outflow is weak, this phenomenon disappears. Thus, these new results may also cause X-ray variabilities in galactic black holes sources and optical/UV variabilities in Active Galaxies and Quasars. A preliminary report on various shock instabilities are presented in Molteni et al. (2001).

In the next Section, we present the formulation of the problem in both Smoothed Particle Hydrodynamics (SPH) and in TVD method. In $\S 3$, we present the numerical simulation results and demonstrate the instabilities. In $\S 4$, we discuss our results in light of shear instability. Finally, in $\S 5$, we draw conclusions.

\section{Formulation of the Problem}

It has been discussed quite extensively in the recent literature that matter close to a black hole must be sub-Keplerian (e.g., Chakrabarti, 1996). This is because the boundary condition on the horizon forces the flow to radially move with the velocity of light. Thus, matter moving in Keplerian disks farther out must become sub-Keplerian as it approaches a black hole. (In wind accreting objects, accreting matter could be sub-Keplerian throughout.) This assumption is in vogue in the literature in the context of other flow models (cf. Narayan \& Yi 1995, Narayan, Kato and Honma, 1997, Artemova et al. 2001; See, however Armitage et al., 2001 and Hawley, 2000, for recent treatment of the physical conditions of canonical disks in the zone of the last stable orbit.) In the simulations described below, we start with a sub-Keplerian flow. For convenience, we use $r_{g}=2 G M_{B H} / c^{2}$ the Schwarzschild radius of the black hole, to be the unit of distance, $r_{g} / c$ to be the unit of time and $\rho_{r e f}$, a reference density to be the unit of density of matter. Here, $G$ and $c$ are the universal gravitational constant and velocity of light respectively and $M_{B H}$ is the mass of the black hole. We use Paczyński-Wiita (1980) pseudo-potential to describe gravitational field around a Schwarzschild black hole. In all our simulations, we use axisymmetry and flow both above and below the equatorial plane. The first assumption is justifiable, especially because the black hole itself is strictly axisymmetric and inner boundary condition forces the flow to be axisymmetric close to the hole. However, far away, our assumption restricts us from studying non-axisymmetric nature of this instability. This can be overcome only with three-D simulations we plan to perform in future. We inject matter at the outer boundary symmetrically with respect to the equatorial plane. We supply the sound speed, radial velocity, density and specific angular momen- 
tum at the outer boundary. We use two numerical procedures, namely, Smoothed Particle Hydrodynamics (Monaghan 1985; MSC96) and TVD method (a combined Lax-Friedrichs-Osher scheme suggested by Vyaznikov, Tishkin, \& Favorsky, 1989). For a subKeplerian flow at a distance of $r=X$, the free-fall time scale is given by $t_{f f}=X^{3 / 2}$. Thus if the outer boundary where matter is injected is kept at $X=50$, one obtains $t_{f f} \sim 350$. For a convincing result, the simulation must run at least ten times this time scale. We included bremsstrahlung cooling in the hydrodynamic code however we verified that the instability we discuss is not due to any cooling processes. The code were tested with theoretical solutions. For instance, in Molteni, Ryu \& Chakrabarti (1996) the codes were found to produce nonlinear features such as standing shocks roughly when theoretical simulations predict them.

\section{Results}

All the simulations presented here are carried out with an ideal gas of polytropic index $\gamma=5 / 3$ and a black hole of mass $M_{B H}=10^{8} M_{\odot}$. Fig. 1 shows the results of the $\mathrm{SPH}$ method at four different times $t=3000,5000,7500$, and 13000 (time marked in each box). Each panel shows velocity vectors in $X-Z$ (meridional) plane, $Z=0$ being the equatorial plane. To bring clarity, only one out of three arrows have been plotted (this is why there are 'gaps', especially in the inflow area). The inflow parameters are: radial velocity $v_{r}=-0.1240$, sound speed is 0.0249 , $\rho_{\text {ref }}=1.25 \times 10^{-19}$ and $\lambda=1.95$ is the specific angular momentum. This $\rho_{\text {ref }}$ corresponds to an accretion rate of $\dot{M}=1.25 \times 10^{-4} \dot{M}_{E d d}$. We also include bremsstrahlung cooling process. This tends to cool matter whenever it becomes denser and hotter. As a result, more matter 'sticks' together and produce enhanced clumpiness. Other than this, the bending instability is produced even without cooling.

There are several features which are to be noted. First, matter is found to be repeatedly 'pinched' during accretion process at a regular distance of $2-3 h(r)$, where $h(r)$ is the local height of the inflow. Second, matter bounces back from the centrifugal barrier and generates an outflow (outward pointing arrows) part of which falls back on the disk and deposits energy and momentum on the disk. Third, accretion flow bends very severely and often the amplitude of the bending wave exceeds ten Schwarzschild radius, at a distance of $10-20 r_{g}$. Fourth, the flow switches upper-half to lower-half and vice-versa in a time scale of about $t_{s w}=10000$ in units of the light crossing time of the black hole. For $M_{B H}=10^{8} M_{\odot}$, this corresponds to a switching time of about four months! For a galactic black hole of $M_{B H}=10 M_{\odot}$ this time scale would be exactly 1 second. While some of these features may be enhanced because of our finite location of the boundary and also because we assume strictly axisymmetric system, we believe that general behavior should be similar in a threedimensional simulations as well. Vortices produced asymmetrically are found to affect the outflow rates in upper and lower halves. As a result, the momentum flux in the jet itself may be quasi-periodically switched to high and low values.

In Fig. 2, we use the TVD method. Here we inject matter at $r=500 r_{g}$ and use radial velocity $v_{r}=-0.0444$, sound speed is $2.83 \times 10^{-3}$, specific angular momentum is $\lambda=1.90$ and reference density is $\rho_{\text {ref }}=10^{-19}$. We use $129 \times 129$ unequally spaced grids. Velocity field is shown with arrows drawn at every alternate grids for clarity, except in regions $|x|<100$ and $|z|<100$ where fewer arrows are drawn. Superposed on the velocity field are contours of constant density, the outermost one closest to the axis (or the uppermost/lowermost ones at the outer boundary) being that of $\rho=0.25 \rho_{\text {ref }}$. Density in each contour inward is increased by a factor of two. Disk is found to be bent at several regions by a different degree.

We plot in Fig. 3 the total amount of matter in the upper (solid) and the lower (dashed) halves as a function of time (in units of $r_{g} / c$ ). Masses in the two halves vary in a complementary way. Large scale fluctuations are found in all time scales, ranging from 1000 to 100,000 time units or more (certainly the largest time-scale is related to the location of the boundary). In Fig. 4, we show the variations of density (solid curves, in units of $\rho_{\text {ref }}$ ) and radial velocity (dashed curves, in units of velocity of light) along the $Z$ - direction of the flow at three radial distanced $X=50,150$ and 300 (marked) respectively. Radial velocity has been multiplied by a factor of 30 to bring the plots in the same scale. While quantities at $X=300$ are more or less symmetric with respect to the equatorial plane $(Z=0)$, quantities at $X=150$ have peaks in density and velocity in the upper-half $(Z>0)$ while those at $X=50$ have peaks in density and velocity in the lower-half $(Z<0)$. The velocity 
jumps sharply from negative values (inflow) to positive values (outflow). Thus a very strong shear is operating on the surface of the disk. The effects of this are discussed below.

\section{Possible Origin of Bending of the Accre- tion Flow}

A particle of specific angular momentum $\lambda$ is bounced off a centrifugal barrier located at $r$ where the centrifugal force balances gravity. A flow has a pressure and therefore it bounces farther out. Outflowing wind and the incoming accretion interact and shear instability could be developed. Chandrasekhar (1961, $\S 105)$ provides a dispersion relation for a uniformly rotating flow of angular velocity $\Omega$ (angular momentum vector pointing in the $Z$ direction) streaming with velocities $v_{1}$ and $v_{2}$ along $\mathrm{X}$-axis. For $\Omega=0$ this relation gives the perturbation frequency $\omega$ as a function of wavenumber $k$ to be: $\omega=-k\left(v_{1}+v_{2}\right) / 2 \pm$ $\left[-k_{x}^{2}\left(v_{1}-v_{2}\right)^{2} / 4\right]^{1 / 2}$ where we assumed density to be equal in both the media. The perturbation grows in time scale of,

$$
t_{K H}=\frac{2 \pi}{\omega_{i}}=\frac{4 \pi}{k\left(v_{1}-v_{2}\right)}=\frac{2 \lambda}{v_{1}-v_{2}},
$$

where, $\omega_{i}$ is the imaginary component of $\omega$ and $\lambda=$ $2 \pi / k$ is the radial wave length.

From Figure 4, one can easily compute the growth time scale at various locations. For instance, at $x=300,150$ and 50, for perturbation lengths comparable to the local width of the disk, $\lambda_{x} \sim 80,60$ and 40 , and velocity differences $v_{1}-v_{2} \sim 0.13,0.17$ and 0.25 respectively, growth time scales are $t_{K H} \sim$ 5000,1850 and 350 respectively. For a black hole of $M_{B H}=10^{8} M_{\odot}$ as we are considering here, these time scales correspond to $14,8.3$ and 3.5 days respectively. The simulations were carried out till $t=220,000$ and is much larger than the above time scales. For galactic black hole candidates $\left(M=10 M_{\odot}\right)$, the times scales would be $0.52 \mathrm{~s}, 0.185 \mathrm{~s}$ and $0.035 \mathrm{~s}$ respectively and oscillations of radiations would have frequencies $\sim 2 \mathrm{~Hz}$, $5.4 \mathrm{~Hz}$ and $28.5 \mathrm{~Hz}$ respectively. Thus the effect of the oscillation is likely to produce a power spectra with excess power in around $2-30 \mathrm{~Hz}$. We note that for low $\lambda, v_{1}$ is large but the wind is absent. For high $\lambda, v_{1}$ is smaller, but $v_{2}$ is large. So the frequency should be a function of the input parameters. When rotation is present, $\omega$ is obtained not by analytical means, but by using the method of characteristics (Chandrasekhar,
1961). When the axisymmetry is removed we still expect such instabilities, perhaps spiral in nature.

\section{Discussions and Conclusions}

We showed that in presence of outflows, there is a significant degree of time-varying distortion of the accretion disk surface. The interaction of the oppositely moving flows suggest that the distortion or bending is mainly due to the shear instability, although local heating effects, momentum deposition of the outgoing subsonic flow onto the disk, and local rotational effects may not be ruled out. Disk distortion is found to be a generic phenomenon provided both the disk and the jet form and is present in all length scales whenever shear effects are important whether cooling is present or not. We found that both Smoothed Particle Hydrodynamics and TVD Methods show this instability. The effects we discuss are very similar to what Norman, Winkler \& Smith (1982) and Chakrabarti (1988) discussed in the context of instabilities in jets in presence of terminal Mach shock. There too back-flowing cocoon formed around a jet has a destabilizing effect on the jet. In the case of jets, the back-flow is due to tangential discontinuity separating the jet matter from that of the ambient matter. In the present case, the back-flow is due to the centrifugal barrier.

Observationally we expect that such a behaviour is likely to produce time variabilities in radiation emitted from the inner part (say, $r<200-300 r_{g}$ ) of the disk. Thus whereas in AGNs, optical and UV radiations could be seen in time scales of days, in galactic black hole candidates, soft and hard X-rays are likely to show variabilities in time scales of seconds or less. Since the whole disk does not oscillate coherently or quasi-coherently, the power density spectrum is likely to produce a very broad bump due to this type of oscillations.

\section{REFERENCES}

Armitage P. J., Reynolds C. S., Chiang J., 2001, ApJ 548,868

Chakrabarti, S.K. 1988, MNRAS 235, 35

Chakrabarti, S.K. 1996, ApJ, 464, 664

Chandrasekhar, S., 1961, Hydrodynamic and Hydromagnetic Stability, Oxford University Press, New York 
Eggum, G.E., Coroniti, F.V. \& Katz, J. 1987, ApJ, 323,634

Greenberg, R. \& Brahic, A. 1984, Planetary Rings, University of Arizona press, Tucson

Hawley J. F., 2000, ApJ 528, 462.

Hawley, J.F., Wilson, J.W. \& Smarr, L.L. 1984, ApJ, 277,296

Igumenshchev, I., Abramowicz, M., Narayan, R. 2000, ApJ 537, L27

Monaghan J.J., 1985, Comp. Phys. Repts., 3, 71

Molteni, D., Lanzafame, G. \& Chakrabarti, 1994, ApJ 425, 161

Molteni, D., Sponholz, H. \& Chakrabarti, S.K. 1996, ApJ 457, 805

Molteni, D., Valenza, M.A., Gerardi, G., Chakrabarti, S.K. \& Acharya, K., 2001, Proceedings of the Ninth Marcel Grossman Meeting (Ed.) R. Ruffini, World Scientific Press, Singapore (in press)

Narayan, R., Yi, I.,, 1995, ApJ, 444, 231

Narayan, R., Kato S. and Honma F., 1997, ApJ, 476, 49

Artemova, I. V.; Bisnovatyi-Kogan, G. S., Igumenshchev, I. V., Novikov, I. D., ApJ, 2001, 549, 1050

Norman, M.L., Winkler, K.-H. A \& Smith, M.D., 1982 A\&A 113, 285

Paczyński, B. \& Wiita, P.J., 1980, A\&A, 88, 23

Pringle, J. 1981, ARA\&A, 19,137

Pringle, J. 1997, MNRAS 292, 136

Ryu, D., Chakrabarti \& Molteni, D. 1997, ApJ 474, 378

Sawada, K., Matsuda, T., \& Hachisu, I. 1986, MNRAS 219, 75

Vyaznikov, K. V., Tishkin, V. F., \& Favorsky, A. P. 1989, Mathematical Modeling, 1, 79

This 2-column preprint was prepared with the AAS IATEX macros v3.0.
Fig. 1.- Examples of the oscillation of the disk around the equatorial plane at different times of SPH simulation. The meridional planes $(\mathrm{X}-\mathrm{Z})$ are shown. Distances are measured in units of $r_{g}$ and the times are measured in units of $r_{g} / c$. The interaction between the outflow and the inflow is clear in these pictures. One out of three arrows have been plotted for clarity. See text for parameters.

Fig. 2.- Contours of constant density superposed on the velocity field for a case where injection of matter takes place at $x=500$. Finite Element method has been used. Alternate arrows are plotted for clarity except in regions $|x|<100$ and $|y|<100$ where number of arrows drawn are reduced further. Bending of the disk takes place on all scales.

Fig. 3.- Variation of mass of the matter in the upper and the lower-halves of the simulation for which Fig. 2 was drawn as a function of simulation time. Variation is seen in time scales of 1000 to as large as 100,000 (in geometrical units).

Fig. 4. - Variation of density (solid) and velocity (dashed) at various distance (marked on each curve) from the black hole as a function of the vertical distance $Z$. Note that while the velocity and density are roughly symmetrical around $Z=0$ for $X=300$, they are asymmetrically distributed and $X$ decreases. For $X=150$ peaks in density and velocity lie in the upper-half, and for $X=50$ the peaks lie in the lowerhalf. 


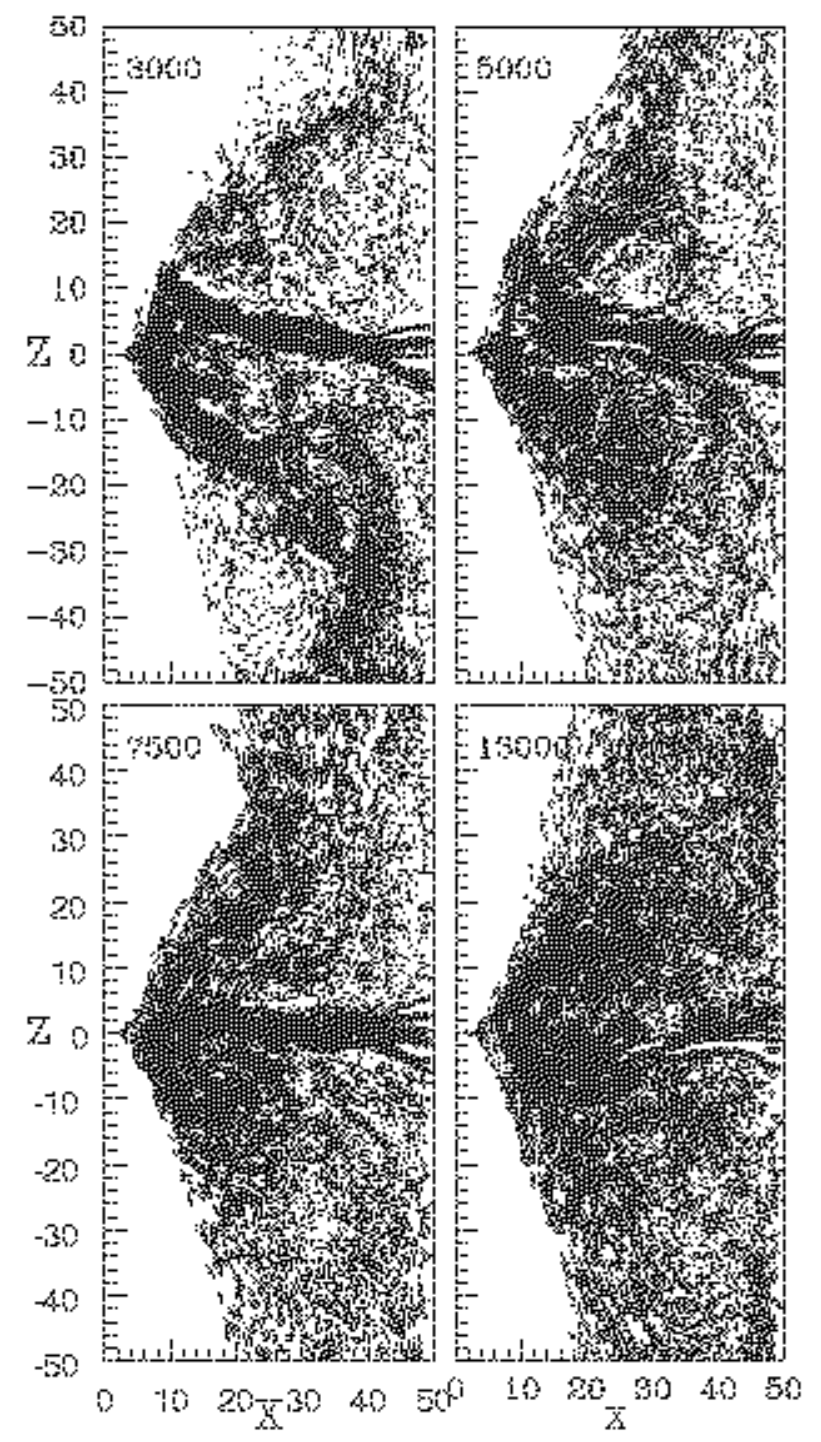




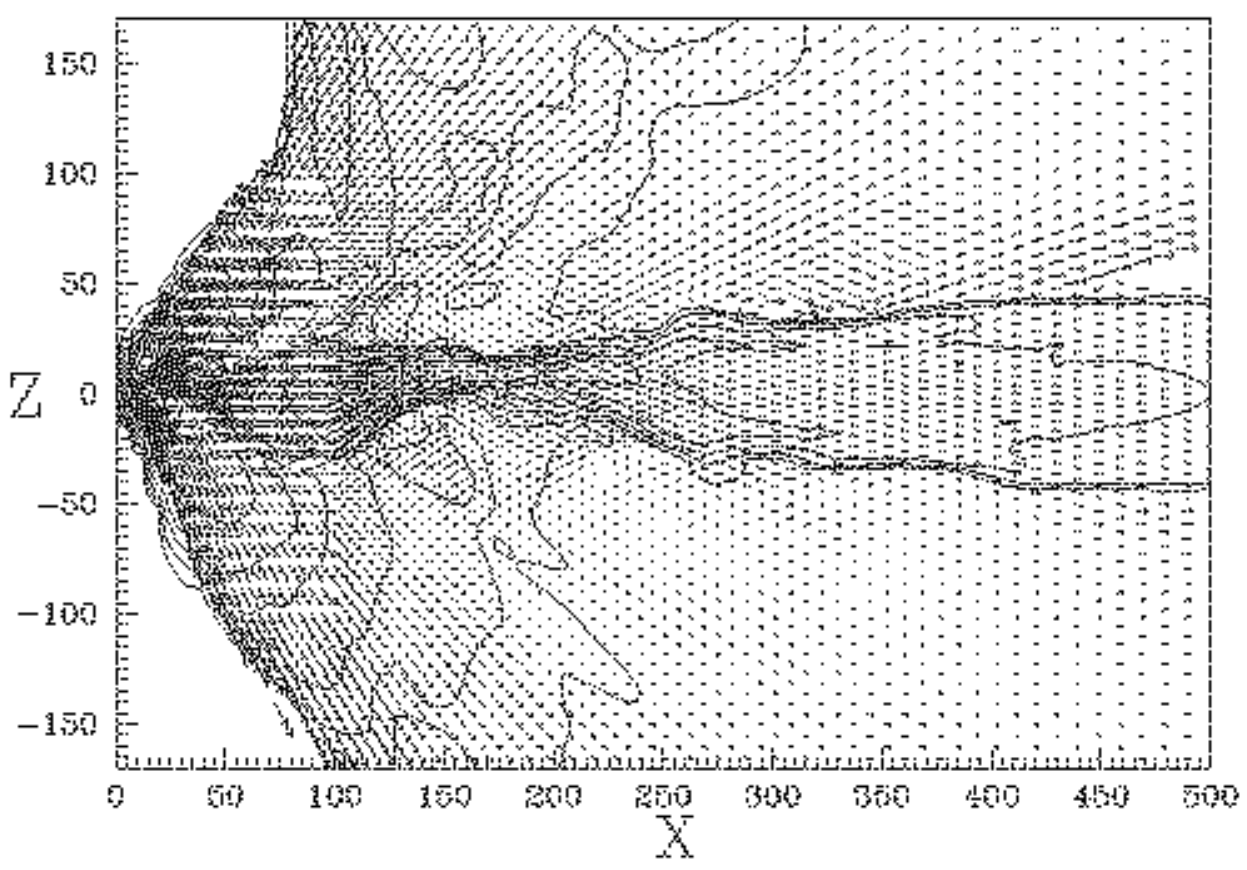




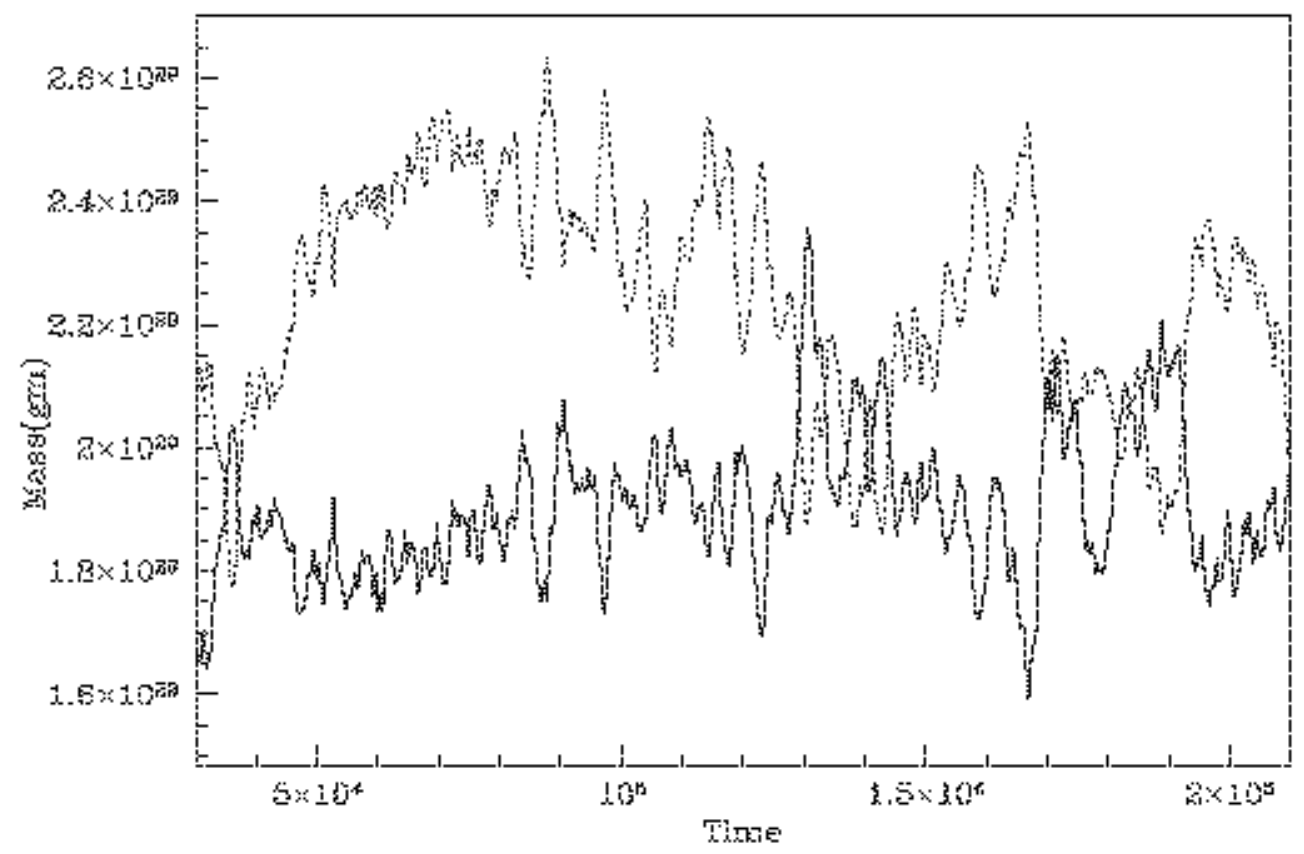




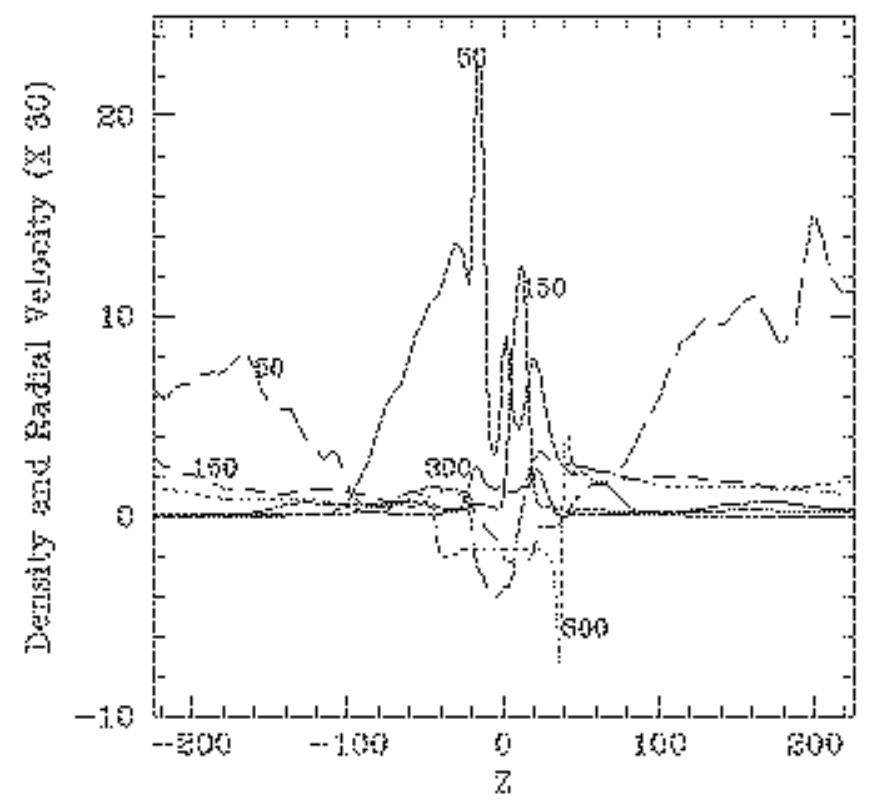

